\title{
Multihop Cellular Networks: Dynamic Channel Assignment and Routing Protocol for Improving Performance
}

\author{
C.P.Maheswaran \\ Assistant professor, Computer Science and \\ Engineering Department \\ Noorul Islam Centre for Higher Education, \\ Tamil Nadu, India.
}

\author{
Bruevin Paul \\ PG Student, Computer Science and Engineering \\ Department \\ Noorul Islam Centre for Higher Education, \\ Tamil Nadu, India.
}

\begin{abstract}
Multihop cellular Networks has the problem of channel assignment when connections are established between the adjacent cells and due to more number of nodes in the path between the sender and the receiver relay delay occurs and cause unwanted delay in the overall end to end communication. To overcome the problems associated with the relay delay and the overall performance in the Multihop Cellular Networks a new protocol is introduced which combines the merits of both the modified Minimum Slot Waiting First Algorithm and the modified Distance Vector Routing. Through the proposed new protocol, we can provide solution for the problem of routing and relay delay in Multihop Cellular Networks and the performance of the packet transmission can be improved.
\end{abstract}

\section{Keywords}

Cellular network, MSWF, CSMA, OCA, CARD, channel assignment.

\section{INTRODUCTION}

Wireless network refers to any type of computer network that is not connected by cables of any kind. The costly process of introducing cables into a building, or as a connection between various equipment locations can be avoided with wireless networks. The wireless communication revolution has brought fundamental changes to data networking, telecommunication and has made integrated networks a reality. By freeing the user from the cord, personal communications networks, wireless LAN's, mobile radio networks and cellular systems harbor the promise of fully distributed mobile computing and communications anytime, anywhere. In case of wireless communication, the sender can be connected to the receiver through a fixed base station, this type of communication is called Single hop Cellular Networks (SCN).

Due to the advancement in the Cellular technology, high speed, high quality data transmission is made possible. Efficient utilization of the radio channel resource is therefore very important because of the increasing number of the mobile subscribers. An efficient routing and a channel allocation algorithm can solve the call and the throughput problem existing in wireless networks. Splitting the cells into smaller cells is simple a way to overcome the draw backs of cellular capacity in the wireless communication. But this technique is costly in terms of the infrastructure and administration. By splitting the cell into more number of smaller cells, the data packets reach the destination through intermediate nodes or the relay nodes, this type of Network is called the Multihop Cellular Networks (MCN). Several recent proposals [5], [7], [8], [11] and [12] look at multihop cellular networks (MCNs), which are designed based on the idea of using mobile and/or fixed terminals to relay signals from source nodes to BSs or destination nodes. The benefit of conventional Single hop Cellular Networks (SCN) is preserved by $\mathrm{MCN}$, where the service Infrastructure is constructed by fixed bases, and it also incorporates the flexibility of ad-hoc networks where wireless transmission through mobile stations in multiple hops is allowed. MCN can reduce the required number of bases or improve the throughput performance, while limiting path vulnerability encountered in ad hoc networks. Thus the multihop cellular networks helps to overcome the problem of hotspot and dead spot problem. Even though the MCN provides a solution to the dead spot and the hotspot problem, it has a disadvantage of channel allocation and multihoping causes delay in transmission of data. The dynamic channel allocation in the multihop cellular networks offers more flexibility and improved capacity. By adopting an efficient channel allocation scheme or a better routing algorithm, the delay in allocating the channel can be reduced.

\section{LITRATURE SURVAIED}

In reference [3] a solution to the main drawback of the relay delay in the Multihop Cellular Network has been proposed. The Multihop Cellular Networks suffers from the relay delay that occurs when the data packets travel from more than one node. A channel allocation algorithm called Minimum Slot Waiting Time algorithm is used to allocate the data packets to the available time slot that comes first. By using the MSWF algorithm the delay that occurs in the multihop cellular networks can be reduced and the overall performance of the transmission can be improved.

In reference [6] the genetic algorithm is provided as a solution to the problem of channel assignment and the algorithm is used to maximize the frequency channel reuse among the cells in the cellular Networks. Channels can be reused among the cells as long as the distance among those cells is sufficiently large. The task is to allocate a minimum number of frequencies to every requested call such that interference constraint is satisfied.

In reference [4] the important goal of the Network is to provide reliable end to end packet delivery in order to achieve this, the data packets are transmitted through multiple nodes. Thus a packet can take multipath route to reach the destination. Through this process the problems of single hop cellular networks can be solved. Thus the Multihop cellular networks are used to provide more reliability to the packet delivery than the single hop cellular networks. 
In reference [9], a clustered $\mathrm{MCN}$ (cMCN) with a fixed channel assignment is proposed. In $\mathrm{cMCN}$, a macrocell is divided into seven microcells, one center microcell, and six virtual microcells around the center microcell. Each microcell is allocated a fixed set of channels. Each virtual microcell has a wireless dedicated information port (DIP) to assign channel and select relaying path and relay stations for mobile nodes. As the fixed channel assignment cannot cope with temporal changes in the traffic pattern, the authors proposed the multihop dynamic channel assignment (MDCA) [10], where channels are assigned to mobile nodes based on the interference condition of the channels in a cell with respect to the other interfering cells. The idea is to maximize the channel availability of the system. Results show that MDCA has performed better than the fixed channel assignment in terms of system capacity.

In reference [1] poor communications to and from the base station due to severe multi-path fading and/or noise can be avoided when multihop relaying is used as an overlay architecture for single hop Time Division Duplex (TDD) and Wideband-Code Division Multiple Access (W-CDMA) cellular networks. Relaying is a fundamental concept in multihop wireless ad hoc networks whereby communications between mobile terminals are carried out through intermediate nodes. The small distance and the potential high transmission rate between the relaying entities draws our attention to the significance of multi-hop relaying in hot and/or dead spots. A major challenge pertaining to the introduction of this technology into cellular networks is the design of an efficient slot assignment algorithm for relaying nodes. A heuristic slot assignment scheme, namely Delay-Sensitive Slot Assignment (DSSA) is proposed to allocate the channel assignment. DSSA is capable of fully utilizing a limited number of channels to enhance spatial reuse and reduce the end-to-end delay.

A dynamic channel allocation algorithm is proposed to dynamically allocate the channels in the Multihop cellular networks and to solve the problem of dynamic channel allocation in the Multihop cellular networks. The MDCA (Multihop Dynamic Channel Assignment) algorithm assigns channels to calls based on interference information in surrounding cells, provided by the Interference Information Table (IIT) in the network. Two different channel searching strategies, Sequential Channel Searching (SCS) and Packing based Channel Searching (PCS), for use in MDCA is studied. A channel reassignment procedure to further enhance the performance is also investigated. MDCA significantly improves the system capacity. Furthermore, the MDCA can efficiently alleviate the call blocking in hot-spot cells.

An architecture called integrated Cellular and Ad hoc Relaying systems (iCAR) was proposed to solve the problem of allocating the channel for the mobile nodes. The proposed iCAR is a new wireless system architecture based on the integration of cellular and modern ad hoc relaying technologies. It addresses the congestion problem due to unbalanced traffic in a cellular system and provides interoperability for heterogeneous networks. This system can efficiently balance traffic loads between cells by using ad hoc relaying stations (ARS) to relay traffic from one cell to another dynamically. This increases the system's capacity cost effectively, and reduces transmission power for mobile hosts and extends system coverage. With a limited number of ARSs and some increase in the signaling overhead (as well as hardware complexity), the call blocking/dropping probability in a congested cell and the overall system can be reduced.

\section{CHANNEL DESIGNING MODEL}

Let us Consider Maximum interface degree of any link is the Network interface degree, where the interference degree of any link $(\mathrm{u}, \mathrm{v})$ is the number of link that interfere with $(\mathrm{u}, \mathrm{v})$ but not with each other. Network interface degree can also be termed as the size of maximum independent set in the sub graph induced by the neighbors of any vertex in the conflict graph. Network interface model is independent of the network topology and is dependent on the interference model. A certain number of time slot is specified for the link schedule. For each time slot, a set of multi-set active links (with a channel assigned to each link) is specified.

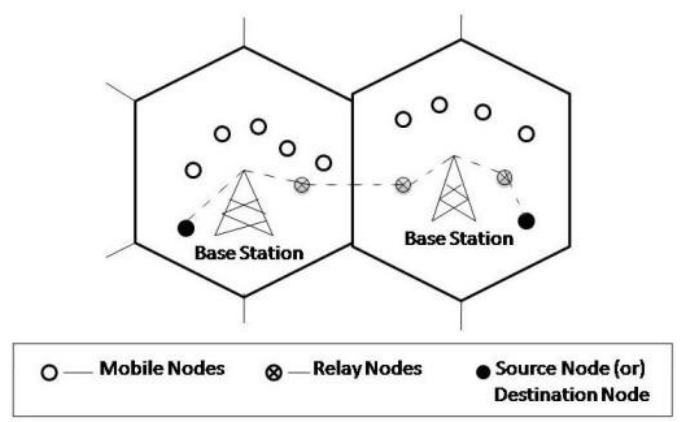

Fig 1: Topology of a MCN environment.

The two main constraints for the valid link schedule are: 1) the active link instances should not interfere. 2) the number of different channels incident on any node $u$ in a time slot is less than v. A call can also be regarded as a session, traffic flow, or a connection. For a connection the nodes on the path is assigned with separate nodes. We define a virtual path called source point $\mathrm{s}$, to represent a initial point of connection in the source node. Separate source points are used in the source node, for different connections. The connection is established from the source to the destination through intermediate nodes called relay nodes. A basic problem in such network topology that involves the relay nodes is the relay delay and the selection of the path from the source to the destination. To reduce the delay, an effective channel assignment is needed. It proposes an optimal and heuristic channel assignment scheme called OCA and minimum time slot waiting first (MSWF) respectively, for a time division duplex (TDD) and wideband code division multiple access (W-CDMA) MCN. Fig 1 gives the general topology of the Multihop Cellular Networks with each cell contain a Base Station, one or more source or destination nodes and few mobile and intermediate nodes.

OCA provides an optimal solution in minimizing packet delay and can be used as unbiased or benchmark tool for comparison among different network conditions or networking schemes.

- We start with a set of relaying paths from source to destination provided by the routing algorithm.

- The set of virtual points denoted as V, determine the relaying paths such that no two paths intersect except at the BS.

- The set of source points is denoted as S, and the set of relaying points as $\mathrm{R}$.

- The set V may correspond to the same physical node.

The routing algorithm decides the relay nodes from the source to the destination. 


\section{MINIMUM SLOT WAITING FIRST ALGORITHM}

MSWF scheme is based on the design goals described before. The Channel assignment scheme resides in the network controller and is also executed in the network controller, such as the radio network control (RNC) in the 3G UMTS. A number of network BSs are connected to the network controller. In Optimal Channel Assignment, when a relaying path is given the main task is to find a channel assignment to minimize the total packet relaying delay. OCA also assures that no signal collision occurs. Other main function of the OCA is that it checks for channel conflicts. An OCA scheme is formulated as an integer linear program to solve the above problems.

A heuristic channel assignment scheme called the Minimum slot waiting first is used to Minimum Slot Waiting First consists of two phases: the proposing phase and the checking phase. A channel which contributes minimum delay is proposed for the node when assigning a channel on a relaying path. The selected channel is called proposed channel and the node the current node. Based on four rules $a, b, c$ and $d$ the channel is checked for channel conflicts. Rules a and b are used to check co-timeslot conflicts where as rules $\mathrm{c}$ and $\mathrm{d}$ are used for checking co-channel conflicts. The proposed channel is accepted if no rules are violated else the channel is eliminated. The two phases are:

\subsection{Proposing phase:}

A channel that contributes the lowest relay delay is proposed to the current node on the path.

\subsection{Checking phase:}

Rule $a$ : The current node is not receiving on the time slot of the proposed channel.

Rule $b$ : The next hop node is not transmitting on or temporary assigned with the time slot of the proposed channel.

Rule $c$ : Nodes on the other routes having there transmission zones in which the next hop node falls are not transmitting on the proposed channel.

Rule d: Nodes that are in the transmitting zone of the current node are not receiving on the proposed channel.

\section{CARD PROTOCOL}

The CARD Protocol is a combination of modified Distance vector routing and Minimum Slot Waiting First channel allocation algorithm. The CARD protocol consists of Routing phase and Allocation phase. In case of the Routing phase the relay nodes are selected on the basis of the minimum distance between two relay nodes and a path for the data packets to reach the destination node is developed. The Routing phase mostly utilized the modified Distance vector routing, which makes use of distance as a metric for allocating the channel resource to the data packets. The Allocation phase consists of mainly the allocation of the channel as in case of the Minimum Slot Waiting First allocation, the data with high priority is allocated the time slot when many data packets compete for channel allocation.

\subsection{Routing Phase:}

The fixed relay nodes are separated from one another by varying distances between them. When dynamic channel allocation strategies are used for fixed relay nodes and if an effective algorithm is not used to decide the optimum path for the data packets to reach the destination then the path taken by the data packets may be longer and time consuming which affects the overall performance of the communication. The new path selection algorithm is used for preventing such drawbacks of selecting longer paths while allocating channel for the data packets to be transmitted from the source to the destination. The path selection algorithm, Modified Dijkstras forms a table with required relay nodes with the minimum distance to each other. This table is referred to create the relay path which gives information about shortest path between the sender and the receiver. The various paths between the Base Station and the Source or Destination nodes are represented in a Tree structure in Fig 2.

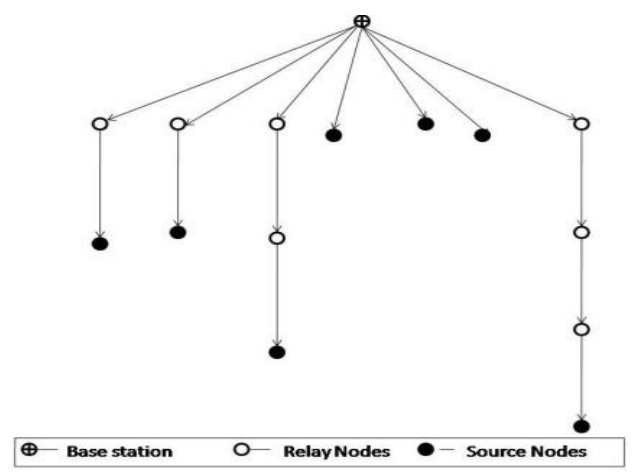

Fig 2: Relay route from Base Station to the source

\subsection{Allocation phase:}

In Allocation phase the channel is allocated to the slot of data. For the allocation of the channel modified Minimum Slot waiting first algorithm is used. After setting the relaying path with the help of the routing phase of the CARD protocol, the next task is to allocate the packets to the suitable channel using the UDF (Urgent Data First) algorithm. The UDF helps to minimize the packet relaying delay and ensure that no signal collision, channel conflict or co-time-slot conflict occurs. It also helps to allocate the slots for the important or the urgent data. Unlike other channel allocation algorithm the UDF is a greedy algorithm which provides a local optimal solution, focusing on minimizing delay for the packets on the path of a new call. 
Table 1. Parameters for Simulation

\begin{tabular}{|c|c|c|c|c|c|c|c|c|c|c|c|c|c|}
\hline & & $\begin{array}{c}\text { BS / } \\
\text { mobile } \\
\text { range }\end{array}$ & $\begin{array}{c}\text { BS / } \\
\text { mobile } \\
\text { capacity }\end{array}$ & $\begin{array}{l}\text { Time } \\
\text { Slots / } \\
\text { frame }\end{array}$ & $\begin{array}{l}\text { Time- } \\
\text { Slots/ } \\
\text { uplink }\end{array}$ & $\begin{array}{l}\text { Codes / } \\
\text { Time } \\
\text { Slots }\end{array}$ & $\begin{array}{c}\text { Max. } \\
\text { hop } \\
\text { count }\end{array}$ & $\begin{array}{c}\text { Data } \\
\text { rate } \\
\text { per code }\end{array}$ & $\begin{array}{l}\text { No. of } \\
\text { source } \\
\text { nodes }\end{array}$ & $\begin{array}{c}\text { Call } \\
\text { request } \\
\text { rates }\end{array}$ & $\begin{array}{c}\begin{array}{c}\text { Call } \\
\text { holding } \\
\text { time }\end{array} \\
\end{array}$ & Antenna & $\begin{array}{c}\text { Simulation } \\
\text { Duration }\end{array}$ \\
\hline Scenario 1 & $\begin{array}{c}\text { OCA/ } \\
\text { MSWF/ } \\
\text { CARD }\end{array}$ & $240 \mathrm{~m}$ & $1040 \mathrm{kbps}$ & 6 & 6 & 6 & 5 & 45.4 kbps & 46 & \multirow{4}{*}{$\begin{array}{c}0.5 \text { calls } / \\
\text { min. }\end{array}$} & \multirow{4}{*}{$1 \mathrm{~min}$} & \multirow{4}{*}{$\begin{array}{c}\text { Directional } \\
\text { antenna with } \\
\text { beam angle } 45^{\circ}\end{array}$} & \multirow{4}{*}{20 mins. } \\
\hline \multirow{3}{*}{ Scenario 2} & OCA & $240 \mathrm{~m}$ & $1040 \mathrm{kbps}$ & 16 & 14 & 6 & 8 & \multirow{3}{*}{$15.13 \mathrm{kbps}$} & \multirow{3}{*}{75} & & & & \\
\hline & MSWF & $380 \mathrm{~m}$ & $830 \mathrm{kbps}$ & 16 & 14 & 5 & 8 & & & & & & \\
\hline & CARD & $1150 \mathrm{~m}$ & $210 \mathrm{kbps}$ & 16 & 14 & 2 & 2 & & & & & & \\
\hline
\end{tabular}

The UDF is designed on the basis of three principles: 1.Eliminate the conflicting channel, by checking the available channel as in case of the checking phase of MSWF, 2.Select channels which contribute minimum relaying delay (time-slot waiting time) and 3.Allocate the packets. The UDF Algorithm in addition to the MSWF algorithm to allocate the channel for the important or urgent data, Urgent data First(UDF) algorithm is used. In case of the UDF, the priority bits are used to specify the urgent or the important data packets and the priority bits are not added to the ordinary data packets. UDF algorithm checks whether the data packet is an ordinary data packet or an urgent one. Once a data packet is identified as an urgent packet, then the channel is allocated to the urgent packet depending on the priority of the packet.

\section{Performance Evaluation}

The performance of CARD is compared with respect to OCA and the MSWF case at different conditions in terms of number of time-slots, number of hop counts, nodal densities, and cell sizes and parameters. The simulation model has a single source and several destinations to allocate the channel. The number of relaying nodes varies from 0 to 150 in increments of 30 .We separate the role of source node and relaying node so that the case in which no mobile nodes are willing to relay signals can be captured. The different number of relaying nodes is used to model different network densities and traffic patterns. All the nodes are uniformly distributed in a circular region with a radius of $1,150 \mathrm{~m}$ centered at the BS. Fig 3 represents the simulation model of a single cell Multihop Network.

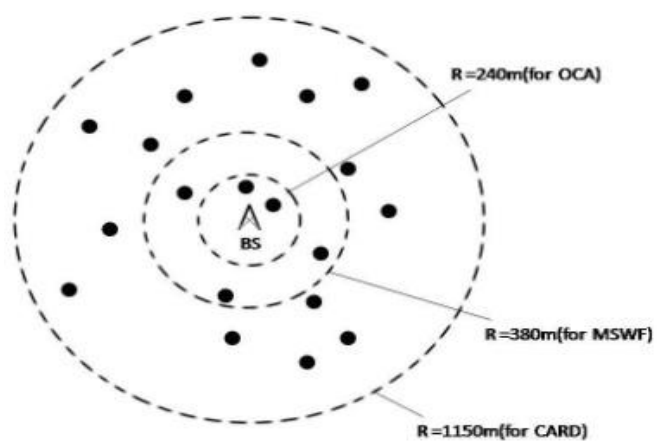

Fig 3 Model of a Single Cell

Scenario 1 (Small number of available channels): In this scenario, the BS and mobile nodes have a transmission range of $250 \mathrm{~m}$ along with a cell capacity of $1,040 \mathrm{kbps}$. Each TDD data transmission frame has a length of 4.33 milliseconds along with 6 time-slots. This frame size is $1 / 3$ of the standard data transmission frame size, which is $12 \mathrm{~ms}$ and 16 timeslots, in the WCDMA standard (Holma \& Toskala, 2004). All 6 time-slots are assigned for the BS uplink transmission. A maximum number of six codes can be assigned to each timeslot. Thus, there are 36 channels (timeslot code pairs). Each code corresponds to a data rate of $45.4 \mathrm{kbps}$, which is 3 times $15.13 \mathrm{kbps}$ (the data rate of one code with spreading factor 16 in the WCDMA standard) (Holma \& Toskala, 2004). Each call uses one code at a constant bit rate. The maximum number of hops is set to 4 to avoid excessive delay. The number of source nodes is 46 , which is larger than 36 . Note that a relatively large number of source nodes are used because a small hop count is used. Thus, even though the network density is high, there are source nodes not able to reach the base stations to use the available channels. In this setting, there is enough demand reaching the BS to establish a congested condition to test the effectiveness of the schemes.

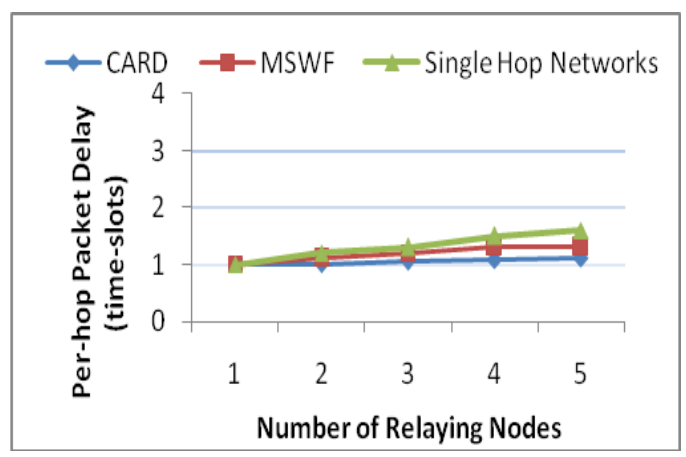

Fig 4: Per-hop Packet delay.

Scenario 2 (Large number of available channels): In this scenario, for OCA case, the transmission ranges (cell size) of the BS are 240 and for MSWF transmission ranges (cell size) of the BS are $380 \mathrm{~m}$ with cell capacity of 1,040 and $830 \mathrm{kbps}$ respectively, whereas the transmission range of mobile nodes is $240 \mathrm{~m}$.

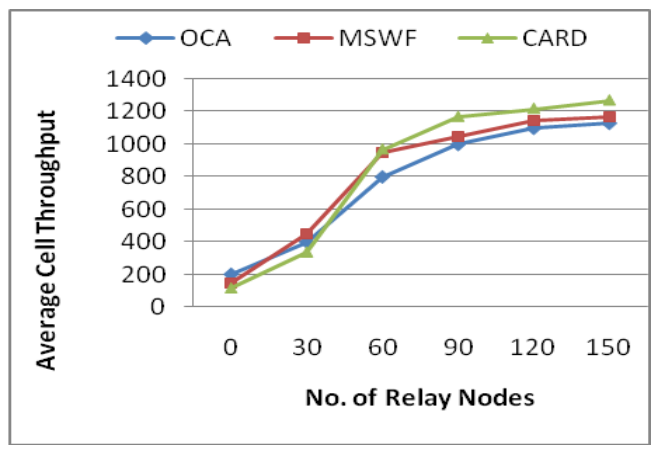

Fig 5: Cell Throughput.

For the CARD case, the transmission range of the BS is 1,150 $\mathrm{m}$ with cell capacity of $210 \mathrm{kbps}$. The data transmission frame 
of each TDD has a length of $10 \mathrm{~ms}$ and has 16 time-slots, according to the WCDMA standard (Holma \& Toskala, 2004).The number of uplink time-slots and the number of downlink time-slots are 14 and 2 respectively. For OCA R240 $\mathrm{m}$ case, each time-slot can be assigned five codes. Thus, there are 65 uplink channels. For MSWF-R390 m case, each timeslot can be assigned with four codes and there are 52 channels. Each code corresponds to a data rate of $15.13 \mathrm{kbps}$ (Holma \& Toskala, 2004). For the CARD case, each time-slot can be assigned with maximum 2 codes in this model; our focus is on channel assignment. We assume there is a routing protocol to provide relaying paths. We assume nodes to be static (or with limited mobility) because we focus on studying the performance of channel assignment schemes. Fig 4 shows the reduction in the Per-hop packet delay when Card protocol is compared with MSWF and Single Hop Networks. Fig 5 compares the cell throughput of CARD protocol, MSWF and OCA algorithms and it shows the increase in throughput when Card protocol is used.

\section{CONCLUSION}

We propose a protocol that uses both channel allocation and routing along with a provision of sending priority data in a TDD W-CDMA or any TDD MCN. The CARD protocol makes use of the modified MSWF algorithm for the channel allocation and the Dijkstras algorithm to set the shortest route between the sender and the receiver. Channel assignment and routing are the components that were analyzed in this paper. Other functions such as mobility management, routing, power control, power efficiency, quality of service management, and security are still need to be addressed. These are interesting topics for future research.

\section{REFERENCES}

[1] M. Al-Riyami, A.M. Safwat, and H.S. Hassanein, "Channel Assignment in MultiHop TDD W-CDMA Cellular Networks," Proc. IEEE Int'l Conf. Comm. (ICC '05), (2005), pp. 1428-1432.

[2] X.J. Li and P.H.J. Chong, (2008) "A Dynamic Channel Assignment Scheme for TDMA-Based Multihop Cellular Networks," IEEE Trans. Wireless Comm., vol. 7, no. 6, pp.1999-2003.
[3] Y. Hung Tam, Robert Benkoczi, Hossam S. Hassanein and Selim G. Akl, "Channel Assignment for Multihop Cellular Networks: Minimum Delay," IEEE Trans. on mobile computing, vol. 9, no. 7, 2002, pp.10221034.

[4] Y.D. Lin and Y.C. Hsu, "Multihop Cellular: A New Architecture for Wireless Communications", Proc. IEEE INFOCOM, (2000), pp. 1273-1282.

[5] G.N. Aggelou and R. Tafazolli, "On the Relaying Capacity of Next Generation GSM Cellular Networks," IEEE Personal Comm., vol. 8, no. 1, (2001), pp. 40-47.

[6] X. Fu, A.G. Bourgeois, P. Fan, and Y. Pan, (2006) "Using a Genetic Algorithm Approach to Solve the Dynamic Channel- Assignment Problem," Int'l J. Mobile Comm., vol. 4, no. 3, pp.333-353.

[7] Sophia Antipolis, Valbbonne, Third Generation Partnership Project (3GPP); Technical Specification Group Radio Access Network, "Opportunity Driven Multiple Access (ODMA)”, (1999) (3G TR 25.924 Version 1.0.0).

[8] S. De, O. Tonguz, H. Wu, and C. Qiao, "Integrated Cellular and Ad Hoc Relay (iCAR) Systems: Pushing the Performance Limits of Conventional Wireless Networks," Proc. IEEE Hawaii Int'l Conf. System Sciences (HICSS '02), (2002) pp. 3899-3906.

[9] X.J. Li and P.H.J. Chong, "Performance Analysis of Multihop Cellular Network with Fixed Channel Assignment," Wireless Networks, vol. 16, Feb. 2010, pp. 511-526.

[10] X.J. Li and P.H.J. Chong, “A Dynamic Channel Assignment Scheme for TDMA-Based Multihop Cellular Networks," IEEE Trans. Wireless Comm., vol. 7, no. 6, (2008), pp. 1999-2003.

[11] Y.D. Lin and Y.C. Hsu, "Multihop Cellular: A New Architecture for Wireless Communications," Proc. IEEE INFOCOM, (2000), pp. 1273-1282.

[12] A.M. Safwat "A-Cell: A Novel Multi-Hop Architecture for $4 \mathrm{G}$ and $4 \mathrm{G}+$ Wireless Networks," Proc. IEEE Vehicular Technology Conf. (VTC '03), (2003), pp. 2931-2935. 